\title{
Book Review. Alex Broom and Katherine Kenny's Survivorship: A Sociology of Cancer in Everyday Life
}

\author{
ÁGNES SÁNTHA \\ Affiliation: Sapientia Hungarian University of Transylvania \\ Faculty of Technical and Human Sciences, Târgu Mureș, Romania \\ Department of Applied Social Sciences \\ Email:santhaagnes@ms.sapientia.ro
}

\begin{abstract}
Cancer has become the second largest cause of death and a central concern in modern societies. Despite increasing survival rates, there is hardly a family that is not directly engaged with the fight against cancer. The brand new book Survivorship: A Sociology of Cancer in Everyday Life (appeared as recently as March 2021) approaches the phenomenon from the the perspective of everyday lives of survivors, their micro-social networks, and health care professionals. Authored by Alex Broom and Katherine Kenny, sociologists from the University of Sydney, and elaborated with a range of qualitative methods, the chapters of the book address issues of social norms, individual tensions of survivors, and emotional approaches to survivorship.
\end{abstract}

Keywords: agency, illness perceptions, cancer, sociology of health, survivorship, normative expectations

Part of the Routledge Studies in the Sociology of Health and IIIness, the book fills a gap in sociology in general and in microsociology, in particular, by completing the medical and quantifying view on cancer with the individual experiences of patients and their networks. The cancer-care industry, and within it, discourse on survivorship, is framed through quantifiable clinical constructions, which show great progress of cancer treatment success in the last decades in the Global North. Disease-centered cancer survivorship, however, misses the person. As an alternative, the person-centered approach offered by this book foregrounds the relational, cultural, economic, and political aspects of illness in everyday life that are outside of the focus of quantifiable encoding. 
Although cancer has always existed and it definitely will remain with us in the foreseeable future, experiences of the illness in late modernity have a particular nuance when life extension is desirable at all costs and mortality is troubling.

The concept of survivorship as an escape postulates some moral qualities of individuals as inherent. Survivors are physically and mentally strong characters, resilient winners who have overcome the disease, and surviving is a moral imperative for the individual in relation to society and the state.

The arguments foreground the processual nature of cancer treatment. The normative trajectory of survivorship is "away from disease": from diagnosis to treatment and then to cure. Stories of survivors in this book present people who live with the illness rather than who have overcome it, some of them without ever being completely cured of it. This is yet another aspect overseen by the disease-centered model that misses the growing number of people living with cancer in the long run.

The book is an example of a qualitative mixed methods approach with data gathered throughout twenty years, using a large number of individual and dyadic interviews and focus group discussions with cancer patients from Australia. Moreover, solicited diaries and photo elicitation of the patients help capture longer-term states of survivors and identify behind-the-scene happenings, broadening the temporal frame and distancing the storyteller from the researcher.

A particularly valuable and interesting chapter of the book develops from the dyadic interviews conducted with patients and their carers. Respondents orient themselves towards their future through a process of negotiation in relation to other people, to the significant others, like spouses, family members, friends, and colleagues. Uncertainty prevails in the narratives of survivors already inherent in medical diagnoses, treatment options, and prognoses themselves. Throughout the chapters, tensions between the normative expectations and the lived experiences of survivors are being discussed. The normative imperative "prepare for the worst, but hope for the best" often preserve various degrees of discontinuity with cancer patients' past, present, and future.

Another tension that prevails in the narratives lies between the clinical diagnosis of terminal cancer and feeling well despite this diagnosis. Deviations from the normative assumptions connected to the "sick role" (Parsons, 1951) that assumes a decline before dying confers uncertainty. 
Similarly, the dominant discourse about survivorship is the positive activity. Cancer patients experience the expectation of being hopeful and fighting a battle while storytellers recount being strong for others, primarily for their families. Demands of attitudes towards survivors are relational in their nature. The normativity of the appropriate disposition is created and reinforced throughout the illness process, creating constant pressure concerning proper behavior.

A further tension originates in the dichotomy of waiting for future clinical updates and, at the same time, trying to live in the present. The omnipresence of disease in the background and everyday life is often experienced as a form of exile, discontinuous from the path of life itself, from the past and the future. The contradiction between entanglement and estrangement brings about the porosity of borders, particularly between time planes that are supposed to be continuous in the normal life course. Yet, when living with cancer, past and future are constantly renegotiated. Clinical prognoses of the survival chances and the time left to live not only crystallize the sense of liminality but are also omnipresent as unsettling factors about the future, thus, they confer ambivalence to the present, too.

Unlike the usual approaches to cancer survivorship, stories of cancer patients in this book depart from the effects of emotions (Fox, 2015) and do not celebrate the phenomenon as an individual triumph independent from relational others, but rather as a form of sociality infused not only with effects but also with morality. Survivorship is a culturally shaped collective act; in other words, it is surrounded by normative expectations about the appropriate emotions, most commonly a will to live, bravery, hope, and positivity (Ehrenreich, 2009; Steinberg, 2015). It is being deployed as a moral commitment and a coercive cultural form, imposing pressure on cancer patients, particularly on those who experience feelings of dread, despair, and capitulation and are thus socially misrecognized. Due to the dominant paradigm of positivism in survivorship, such non-normative negative feelings are often individualized and marginalized.

Within the context of the rise of precision medicine in cancer care and the euphoria associated with it, healthcare professionals' (oncologists', trainees', and nurses') accounts offer a more nuanced view on this promising innovation. The transformative power of the genomic turn is evident in some types of cancer like melanoma or lung cancer. In the background, however, individual molecular therapeutics is associated with ambiguity around the economic interests behind its emergence (Rushford \& Greengalagh, 2020). 
In the stories of healthcare professionals, enchantment-a mixture of hope, faith, refreshment, and the aspiration of progress-towards-cure-intertwining with the breakthrough of precision medicine is challenged by the uneven progress across cancer types, the disproportionate institutionalization throughout Australian hospitals, as well as by the patients' unequal access to care. The tension between the affective enchantment sustained by the media and the clinical reality featuring social inequity carries the potential of patient misinformation or confusion.

Precision medicine is linked to a series of trials for patients as a part of their cancer treatment procedure, beyond the official scientific investigation. Although it carries the heavy burden of inequity, those patients involved in such experiments perceive participation as a civic duty, sometimes as a parting gift to the community and future patients, and often participate in trials in spite of being critical to the assessment of worth and their conviction that their lives should not be prolonged at any cost. Notwithstanding the objective gains of disease reduction, the quality of their lives is inherently compromised. Hope, too, is a complicated social practice.

The book is a critique of orthodox views on cancer survivorship and reveals the social production of the process. Living with cancer is captured as becoming, in its processual nature. Despite the relatively large potential of agency within the fight for survival, the process entails a strong structural gradient that manifests itself in the reproduction of social inequalities and unequal treatment opportunities for patients. Much more than an individual issue, survivorship becomes a social, economic, and political practice.

\section{References}

Broom, A., \& Kenny, K. (2021). Survivorship: A sociology of cancer in everyday life. Routledge, Taylor and Francis Group.

Ehrenreich, B. (2009). Smile or die: How positive thinking fooled America and the world. Granta.

Fox, N. J. (2015). Emotions, affect and the production of social life. British Journal of Sociology 66(2), 301-318. https://doi.org/10.1111/1468-4446.12119

Parsons, T. (1951). The social system. Free Press.

Rushford, A. \& Greengalagh, T. (2020): Personalized medicine, disruptive innovation, and "Trailblazer" guidelines: Case study and theorization of an unsuccessful change effort. Milbank Quarterly 98(2), 581-617. https://doi.org/10.1111/1468-0009.12455

Steinberg, D. L. (2015). The bad patient: Estranged subjects of the cancer culture. Body \& Society 21(3), 115-143. https://doi.org/10.1177/1357034X15586240 